\title{
The clinical potential of circulating immune cell counts in primary gastric lymphoma
}

\author{
Ayman Haimour ${ }^{1}$, Osama Abu-Shawer ${ }^{2}$, Mohammad Abu-Shawer $^{3}$, Ali Al-Taji ${ }^{4}$, Tamer Altamimi ${ }^{5}$, \\ Razan Mansour ${ }^{6}$, Justin Z. Amarin ${ }^{6}$, Hala Sultan ${ }^{4}$, Maysa Al-Hussaini ${ }^{7}$ \\ ${ }^{1}$ Department of Biochemistry and Microbiology, University of Victoria, Victoria, Canada; ${ }^{2}$ Harvard Medical School, Boston, MA, USA; ${ }^{3}$ Department \\ of Internal Medicine, Istiklal Hospital, Amman, Jordan; ${ }^{4}$ School of Medicine, The University of Jordan, Amman, Jordan; ${ }^{5}$ Internal Medicine \\ Department, Rochester General Hospital, NY, USA; ${ }^{6}$ Office of Scientific Affairs and Research, King Hussein Cancer Center, Amman, Jordan; \\ ${ }^{7}$ Department of Pathology and Laboratory Medicine, King Hussein Cancer Center, Amman, Jordan \\ Contributions: (I) Conception and design: A Haimour, O Abu-Shawer, M Al-Hussaini; (II) Administrative support: M Al-Hussaini; (III) Provision \\ of study materials or patients: M Al-Hussaini, H Sultan, R Mansour, JZ Amarin; (IV) Collection and assembly of data: A Al-Taji, T Altamimi, \\ A Haimour; (V) Data analysis and interpretation: A Haimour, O Abu-Shawer, M Abu-Shawer; (VI) Manuscript writing: All authors; (VII) Final \\ approval of manuscript: All authors. \\ Correspondence to: Maysa Al-Hussaini, MD, FRCPath. Department of Pathology and Laboratory Medicine, King Hussein Cancer Center, Queen \\ Rania Street, Amman 11942, Jordan. Email: mhussaini@khcc.jo.
}

Background: High neutrophil-lymphocyte ratio (NLR) is linked to poor overall survival (OS) in gastrointestinal tract cancers. This study explores the clinical value of NLR, in addition to absolute lymphocyte count (ALC) and other hematologic parameters in association with distant metastases and OS in primary gastric lymphoma (PGL) patients.

Methods: Clinical data of 139 PGL patients who received treatment at King Hussein Cancer Center (KHCC), Amman-Jordan were retrospectively evaluated. Using data from complete blood count (CBC) tests, the following hematologic parameters: absolute neutrophil count (ANC), ALC, absolute eosinophil count (AEC), absolute monocyte count (AMC), NLR, platelet-lymphocyte ratio (PLR), and monocytelymphocyte ratio (MLR) were assessed in association with the following clinical outcomes: presence or absence of baseline distant metastases and OS. We conducted univariate and multivariate analyses assessing the various hematologic parameters in association with distant metastases.

Results: Univariate and multivariate analyses indicated that patients with an elevated NLR $(>3.14)$ displayed more baseline distant metastases compared to patients with a low NLR $(\leq 3.14)$, (P value: 0.02 and 0.018 , respectively). High baseline $\operatorname{ALC}(>1,819 / \mu \mathrm{L})$ was associated with lower baseline distant metastases (P value: 0.04$)$. In the OS analysis, high baseline ANC $(>5,100 / \mu \mathrm{L}), \operatorname{NLR}(>2.75)$, and PLR $(>0.16)$ were associated with poor OS, (P value: $0.027,0.016$, and 0.011 respectively).

Conclusions: High NLR and ALC were associated with baseline distant metastases. High baseline ANC, NLR, and PLR were associated with poor OS. Hematologic parameters might be potentially helpful in assessing and correlating NLR with the response success to treatment in PGL.

Keywords: Primary gastric lymphoma (PGL); neutrophil-lymphocyte ratio (NLR); distant metastases

Submitted Sep 15, 2020. Accepted for publication Jan 08, 2021.

doi: 10.21037/jgo-20-383

View this article at: http://dx.doi.org/10.21037/jgo-20-383

\section{Introduction}

Extra-nodal non-Hodgkin's lymphomas (NHLs) account for $24-48 \%$ of all the lymphomas around the world (1). The gastrointestinal tract is the most common site for extranodal lymphomas (2); with primary gastric lymphoma (PGL) comprising up to $5-8 \%$ of these extranodal neoplasms $(3,4)$. 
It is well known that B-cell lymphomas predominate in the stomach (3), while T-cell lymphoma is a rare encounter $(2,5)$. Gastric lymphomas range, based on histological classification, from indolent low-grade lymphomas, such as lymphoma of mucosa-associated lymphoid tissue (MALT), to aggressive B-cell lymphomas such as high-grade diffuse large B-cell lymphomas (DLBCL) (2). The etiology of PGL is associated with genetic predisposition (6) and constitutive activation of the inflammatory and immunomodulatory NF-kappa-B pathway due to chromosomal translocations, resulting in neoplastic B cell proliferation (7). MALT lymphoma is associated with the pathogenesis of gastric Helicobacter pylori (H. pylori) infection (2), where H. pylori can induce B-cell proliferation as well as the production of pro-inflammatory cytokines in an $H$. pylori strain-host specific manner $(8,9)$.

The alarming symptoms of PGL, such as gastric bleeding and continuous vomiting are infrequent, whereas the milder non-specific clinical symptoms such as gastrointestinal pain and indigestion can be overlooked, resulting in delayed diagnosis and sometimes affecting the prognosis and outcome (5).

Several molecular biomarkers are believed to be involved in PGL progression, including cellular adhesion molecules (CAMs) such as PECAM-1, a potential promoter of metastases reported as a prognostic marker with unfavorable outcome in PGL (10). On the other hand, human leukocyte antigen (HLA)-DR, an MHC II surface protein expressed on antigen presenting cells, was reported as a favorable outcome-associated prognostic marker in PGL (10).

Inflammation mediated by dysregulated levels of circulating immune cells and pro-inflammatory cytokines is associated with tumorigenesis, distant metastases, and overall patient survival in various cancer types $(11,12)$. Nevertheless, the immediate linkage between inflammation and the pathophysiology of metastases is not completely understood. Neutrophil-lymphocyte ratio (NLR), absolute neutrophil count (ANC), absolute lymphocyte count (ALC), absolute eosinophil count (AEC), absolute monocyte count (AMC), platelet-lymphocyte ratio (PLR) in addition to monocyte-lymphocyte ratio (MLR) were shown to have a potential prognostic role in evaluating patients' survival outcome in different tumors $(13,14)$. Of particular interest, high baseline NLR is an independent predictive factor for poor overall survival (OS) outcomes and baseline distant metastases in various cancers $(14,15)$.

The tumor microenvironment is rich in malignant cells and harbors dysregulated levels of pro-inflammatory cytokines and immune cells (16). Here we briefly introduce some of the crucial immune cells, including players from both the innate and adaptive immune response that we investigate in our study. Myeloid-lineage immune cells include neutrophils, monocytes (precursors for macrophages in tissues) and eosinophils, while lymphoidlineage cells include natural killer cells (NK), cytotoxic T cells, T-helper cells and B-cells. Neutrophils comprise most of the tumor microenvironment-associated immune cells, and are therefore key players in regulating tumor metastases $(16,17)$. An elevated count of circulating neutrophils, leading to a high NLR, could promote metastases through releasing pro-inflammatory cytokines and growth factors, such as vascular endothelial growth factor (VEGF) $(17,18)$. Neutrophils can promote tumor proliferation and metastases via recruitment by cytokines, chemokines and matrix metalloproteases (e.g., IL-17, CXCL5 and MMP-9 respectively) $(17,19)$. Opposing pro-tumor neutrophils (N2 phenotype), circulating lymphocytes such as NK cells and $\mathrm{CD}^{+} \mathrm{T}$ cells play an active role in eradicating cancer cells through targeted cytotoxic cell death, which in turn hinders tumor progression (20) and results in a potentially favorable survival outcome in cancer patients. Unlike the antitumor $\mathrm{CD}^{+} \mathrm{T}$ lymphocytes, heterogeneous $\mathrm{CD}^{+} \mathrm{T}$ cells expressing an extensive array of cytokines can have both proand anti-tumor roles in the tumor microenvironment (21). Myeloid-lineage immune cells such as eosinophils (22) and platelets (23) were shown to have proangiogenic properties and could therefore modulate tumor angiogenesis. The heterogeneity of monocytes, which are also of a myeloid lineage, allows them to have both pro- and anti-tumor roles in the tumor microenvironment (24). Such roles include the differentiation of monocytes into pro - tumor tissue macrophages (M2 phenotype) promoting tumor progression and metastases $(24,25)$.

Our goal in this study is to further explore the clinical value of NLR and other hematologic markers (ANC, ALC, AEC, AMC, PLR, MLR) in PGL patients retrospectively. We examined the statistical strength mapping the relationship between high NLR, in addition to other inflammatory cell counts, with the incidence of distant metastases and survival outcomes. We hypothesize that these hematologic parameters can serve as biomarkers to establish if PGL patients are prone to a higher incidence of distant metastases and poor OS. Consequently, these biomarkers-especially NLR — can potentially highlight the need to modify required therapeutic interventions at earlier phases and correlate with the response success to therapy. 
We present this article in accordance with the REMARK reporting checklist (available at http://dx.doi.org/10.21037/ jgo-20-383).

\section{Methods}

\section{Data collection from medical records}

Data of 139 PGL patients who received their treatment at King Hussein Cancer Center (KHCC) between the years 2010 and 2018 were reviewed. The presence or absence of distant metastatic lesions in PGL patients was confirmed using computed tomography (CT) scans, positron emission tomography (PET) in addition to magnetic resonance imaging (MRI). We assessed OS outcome based on the initial date of diagnosis to the date of death and/or the last date of follow-up.

We excluded all primary gastric cancer patients who were receiving glucocorticoid treatments at the stage of collecting complete blood count (CBC) tests. The use of glucocorticoid treatments in PGL patients was anticipated to impact the reported parameters in the study, as glucocorticoid treatments can result in leukocytosis and particularly neutrophilia in our studied patients $(26,27)$.

The immune cell counts' data, obtained through a CBC test with differential white cell counts, were collected from the patients at time of presentation before undergoing any particular treatment (such as chemotherapy or radiation therapy). The equations used to calculate baseline NLR, PLR and MLR were as follows: NLR = ANC/ALC, MLR = $\mathrm{AMC} / \mathrm{ALC}$ and PLR $=$ platelets number/ALC.

\section{Assignment of the NLR cutoff value and mapping its relationship to other statistical parameters}

The receiver operating characteristic (ROC) curve analysis was utilized to determine the finest NLR cut-off value, which subsequently was used to evaluate its relationship with the presence or absence of baseline distant metastases in PGL patients, taking into consideration the highest degree of joint specificity as well as sensitivity. The following parameters: NLR cutoff value, age category of patients, their gender and the reported site of the primary tumor were assessed for potential linkage with the presence or absence of baseline distant metastases at time of presentation. We utilized logistic regression analysis, at both univariate and multivariate aspects, to study the potential linkage between the different aforementioned variables and the incidence of distant metastases in PGL patients. The standard cut-off value to consider statistical significance was set at a $\mathrm{P}$ value of $\leq 0.05$ to be used throughout our reported data.

\section{Analyzing the relationship between peripheral immune cell counts and the presence or absence of distant metastases in PGL patients}

The flow of data analysis was performed at multiple levels in a step-by-step manner. In the initial step, we focused on the relationship between baseline NLR cut-off value with the presence or absence of distant metastases. For the second step, an assessment of the detection of distant metastases in relation to the following immune cell counts: ALC, ANC, AEC, AMC, PLR and MLR was performed.

\section{Mapping the relationship between peripheral immune cell counts and the patients' characteristics}

Transitioning into the third step, we considered if a potential correlation could link detection of baseline distant metastases with the primary gastric patients' characteristics including age, gender and the reported site of the primary tumor.

Moving to the fourth step of analysis, we conducted a collective multivariate evaluation that consisted of the recorded patients' characteristics (gender, age and reported site of the primary tumor in the stomach) with the NLR cut-off value being a dichotomous variable, which was then used to determine potential association with detection of baseline distant metastases.

\section{Evaluating the relationship between peripheral immune cell counts and OS of PGL patients}

The fifth step was focused on survival analysis, where the relationship between NLR and other peripheral immune cell counts (consisting of ALC, ANC, AEC, MLR, AMC and PLR) was assessed with OS of PGL patients. Lastly, we tested the relationship between different categorical values of NLR (as a categorical variable) in relation to presence of distant metastases as well as in the OS analysis part.

\section{Statistical analysis}

A comprehensive examination of the reviewed primary gastric cancer patients' characteristics was conducted. Data 
Table 1 Clinical characteristics and hematologic parameters of primary gastric cancer patients

\begin{tabular}{|c|c|}
\hline Characteristics & $\mathrm{N}(\%)$ or median (mean) \\
\hline \multicolumn{2}{|l|}{ Age group, n (\%) } \\
\hline$>55$ years & $61(43.9)$ \\
\hline$\leq 55$ years & $78(56.1)$ \\
\hline \multicolumn{2}{|l|}{ Gender, n (\%) } \\
\hline Male & $94(67.6)$ \\
\hline Female & $45(32.4)$ \\
\hline \multicolumn{2}{|l|}{ Tumor location, n (\%) } \\
\hline Stomach cardia & $6(4.3)$ \\
\hline Others (body, fundus, pylorus, etc.) & $133(95.7)$ \\
\hline \multicolumn{2}{|l|}{ Distant metastases, n (\%) } \\
\hline Present & $40(28.8)$ \\
\hline Absent & $99(71.2)$ \\
\hline \multicolumn{2}{|l|}{$\begin{array}{l}\text { Hematologic parameters, median } \\
\text { (mean) }\end{array}$} \\
\hline ANC & $5,100(5,887)$ \\
\hline ALC & $1,819(2,136)$ \\
\hline AEC & $147.5(182)$ \\
\hline AMC & $603(684)$ \\
\hline NLR & $2.75(4.68)$ \\
\hline MLR & $0.318(0.46)$ \\
\hline PLR & $0.158(0.25)$ \\
\hline
\end{tabular}

ANC, absolute neutrophil count; ALC, absolute lymphocyte count; AEC, absolute eosinophil count; AMC, absolute monocyte count; NLR, neutrophil-lymphocyte ratio; MLR, monocyte-lymphocyte ratio; PLR, platelet-lymphocyte ratio.

collected, including gender, age category and peripheral immune cell counts were shown as numerical values and percentages. Statistical parameters including average, standard deviation, and range were computed for the data such as age, NLR, ALC and the remaining immune cell counts. Overall, variances in proportions were statistically assessed with either the $\chi^{2}$ test or Fisher's exact test, while for variances in continuous variables, the latter were assessed with either the $t$-test or the Wilcoxon Rank Test (as nonparametric) reliant on the necessary norms and inputs for statistical analysis conducted. Multivariate analyses were conducted for the following parameters: NLR (NLR $>3.14$ $v s . \mathrm{NLR} \leq 3.14)$, age category, gender as well as the reported site of the primary tumor (stomach cardia versus others), for which statistical probability was based on presence of distant metastases operating the logistic regression model.

To approximate OS rates, Kaplan-Meier curves were plotted; further, the log-rank test was utilized to approximate PGL patients' survival durations across varying hematologic parameters. The hazard ratio (HR) derived from the statistical analyses establishes the association between the studied variables and the survival outcome. The approximate survival times were reported as a median value plus $95 \%$ confidence interval $(\mathrm{CI})$ and HR. Statistical analyses and data compilation were conducted using SAS version 9.4 (SAS Institute Inc., Cary, NC, USA).

\section{Ethical statement}

We conducted retrospective analyses of hematologic markers in PGL patients after obtaining approval by the Institutional Review Board (IRB) committee at KHCC (IRB approval number: 18KHCC54) in accordance with the Declaration of Helsinki (as revised in 2013); individual consent for this retrospective analysis was waived.

\section{Results}

Information of 139 PGL patients are presented in Table 1. The PGL patients studied had a median age of 55 years at presentation. The patients had an OS period of 7.1 months. Around two-thirds of the studied cases were males (67.6\% males vs. 32.4\% females). The mean and the median values for NLR were 4.68 and 2.75 respectively. An NLR value of 3.14, obtained using area under the ROC curve (calculated value of 0.616 , Figure S1). This NLR value was set as the cutoff point for assessing NLR's relationship with distant metastases.

Table 2 shows the statistical assessment of potential linkage between circulating immune cell counts with presence or absence of distant metastases. PGL patients with NLR higher than the calculated cutoff value $(>3.14)$ were found to have a higher chance of having distant metastases, at diagnosis stage, as opposed to patients with NLR lower than the calculated cutoff value $(\leq 3.14)$, [P value: 0.02 , odds ratio (OR): 2.4, CI: 1.14-5.10]. In addition, high baseline ALC $(>1,819)$ was associated with a lower incidence of distant metastases as opposed to low baseline ALC $(\leq 1,819)$ (P value: 0.04, OR: 0.46, CI: $0.22-0.98)$. However, baseline ANC $(>5,100 / \mu \mathrm{L})$, AMC $(>603 / \mu \mathrm{L}), \mathrm{AEC}(>147.5), \operatorname{MLR}(>0.32)$, and PLR $(>0.16)$ 
Table 2 The relationship between immune cell counts and the presence or absence of distant metastases

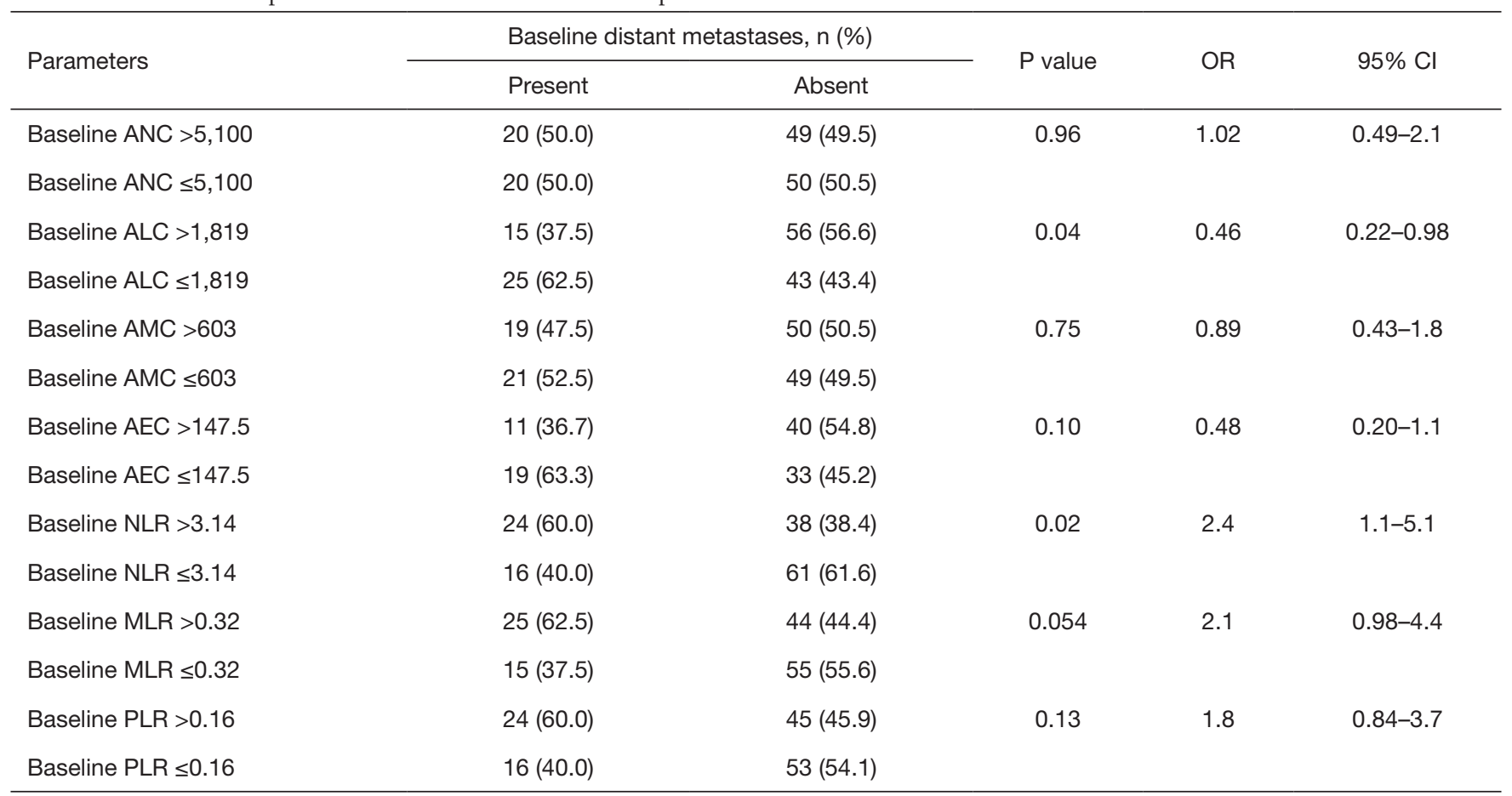

ANC, absolute neutrophil count; ALC, absolute lymphocyte count; AMC, absolute monocyte count; AEC, absolute eosinophil count; NLR, neutrophil-lymphocyte ratio; MLR, monocyte-lymphocyte ratio; PLR, platelet-lymphocyte ratio; OR, odds ratio; $\mathrm{Cl}$, confidence interval.

were not associated with the presence or absence of baseline distant metastases in PGL.

The potential linkage between NLR, and the remaining PGL patients' characteristics, with the presence or absence of baseline distant metastases was examined using both univariate and multivariate analyses (as shown in Table 3). A significant relation of NLR to the presence of baseline distant metastases was found (P value: 0.02, OR: $2.495 \%$ CI: 1.1-5.1). However, age category, gender group and location of the primary tumor: stomach cardia versus other sites (such as body, fundus, pylorus) were not shown to be significantly linked to the presence or absence of distant metastases ( $\mathrm{P}$ value: 0.09, 0.41 and 0.67 respectively). Despite non-significance, but due to their clinical importance, the age category, gender and the reported site of the primary tumor were still included in the multivariate analysis. However, these variables remained insignificant for association with distant metastases in PGL ( $\mathrm{P}$ value: $0.12,0.44$, and 0.50 , respectively). However, NLR remained significantly linked with the chance of having distant metastases in the multivariate analysis $(\mathrm{P}=0.018)$. Furthermore, higher NLR, as a categorical variable, was associated with higher chance of baseline distant metastases as reported in Table 4.

Analysis of the patients' OS are presented in Table 5. Elevated ANC, NLR and PLR were linked to negative OS outcome, (P value: 0.027, 0.016, and 0.011 respectively) (Figures 1-3). As a categorical variable, high NLR was also correlated with negative OS outcome (Table 6). However, baseline AEC, AMC, ALC, and MLR were not shown to be associated with OS in PGL.

\section{Discussion}

To our knowledge, this article emerges as the first study evaluating the clinical value of various immune cell counts, in association with the incidence of baseline distant metastases, in PGL patients. To overcome the limitation of the early non-specific presenting symptoms including pain and indigestion (5), using standard routine tests such as a CBC can better inform if PGL patients might be presenting at an advanced stage; consequently, highlighting the need to modify their required therapeutic interventions, surveillance and consideration of prophylactic strategies at earlier phases.

Throughout this study, we evaluated the potential 
Table 3 Univariate and multivariate analyses for the association of primary gastric cancer patients' clinical characteristics with the presence or absence of baseline distant metastases

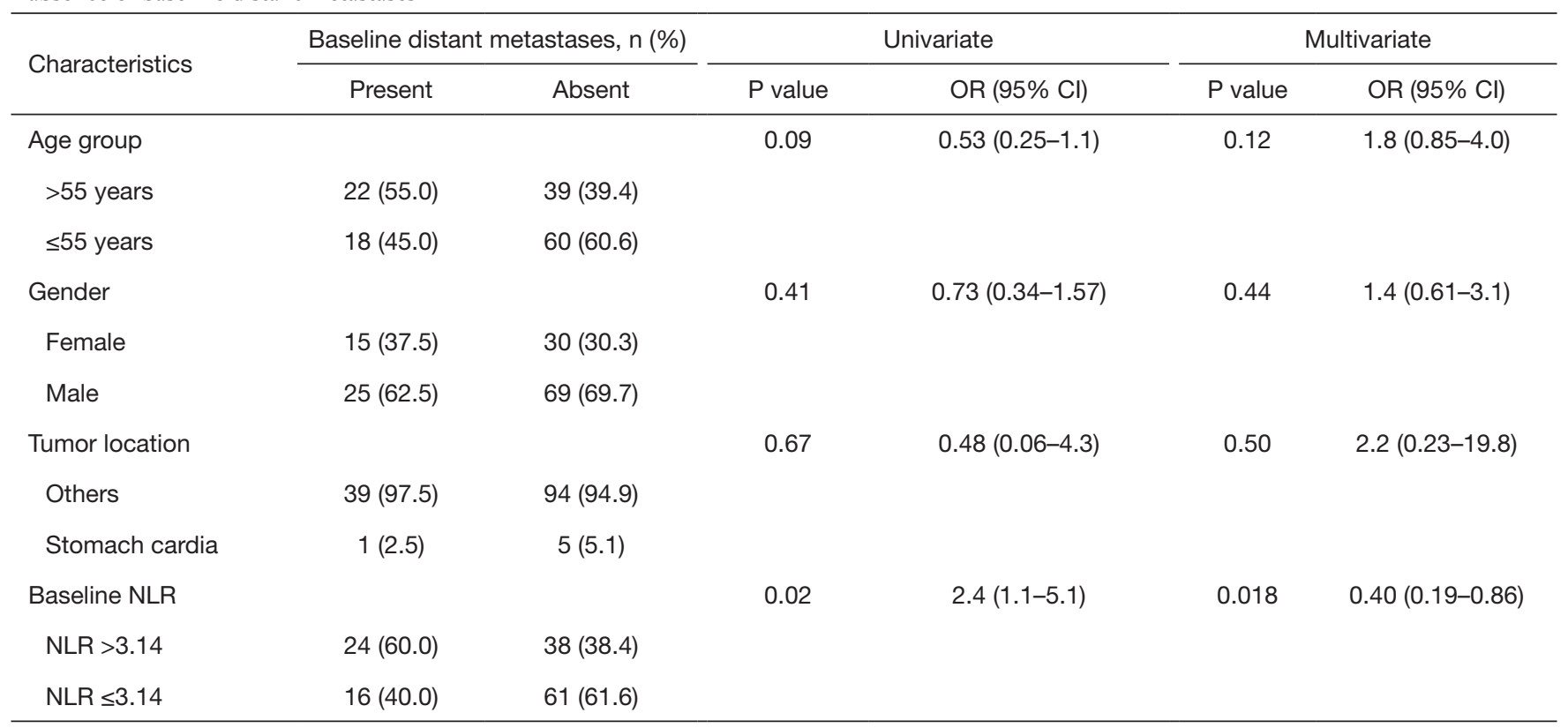

OR, odds ratio; Cl, confidence interval; NLR, neutrophil-lymphocyte ratio.

Table 4 The relationship between NLR, as a categorical variable, and the presence or absence of distant metastases

\begin{tabular}{|c|c|c|c|c|}
\hline NLR & \multicolumn{2}{|c|}{ Baseline distant metastases, $\mathrm{n}(\%)$} & OR & $95 \% \mathrm{Cl}$ \\
\hline$<1.69$ & $7(17.5)$ & $28(28.3)$ & Reference & \\
\hline $1.69-2.75$ & $8(20.0)$ & $28(28.3)$ & 1.1 & $0.37-3.6$ \\
\hline $2.75-4.59$ & $11(27.5)$ & $23(23.2)$ & 1.9 & $0.64-5.7$ \\
\hline
\end{tabular}

NLR, neutrophil-lymphocyte ratio; OR, odds ratio; $\mathrm{Cl}$, confidence interval.

linkage of the following peripheral immune cell counts and ratios: NLR, ALC, ANC, AEC, AMC, PLR and MLR with the incidence of distant metastases and OS outcome in PGL patients. We utilized univariate (Table 2) and multivariate (Table 3) analysis to assess the prognostic value of high baseline NLR in relation to presence of distant metastases in PGL. PGL patients with baseline NLR higher than the cutoff value ( $>3.14)$ were more likely to have distant metastases in both univariate (P value: 0.02 , OR: $2.41, \mathrm{CI}$ : $1.14-5.10)$ and multivariate (P value: 0.018 , OR: 0.40 , CI: 0.19-0.86) analyses.

Furthermore, even as a categorical variable, NLR was also associated with the presence of baseline distant metastases, as higher categorical NLR values indicated a higher incidence of metastases as shown in Table 4.

In addition, high baseline $\mathrm{ALC}(>1,819 / \mu \mathrm{L})$ was associated with lower baseline distant metastases ( $\mathrm{P}$ value: 0.04, OR: 0.46, CI: 0.22-0.98). However, baseline ANC $(>5,100 / \mu \mathrm{L})$, AMC $(>603 / \mu \mathrm{L}), \mathrm{AEC}(>147.5 / \mu \mathrm{L})$, MLR $(>0.32)$, and PLR $(>0.16)$ were not significantly linked to the presence or absence of baseline distant metastases (Table 2).

In the analysis of the patients' OS (Table 5), high baseline ANC $(>5,100 / \mu L)$, NLR $(>2.75)$, and PLR $(>0.16)$ were linked to negative OS outcome, (P value: $0.027,0.016$, and 0.011 respectively) (Figures $1-3$, respectively). In addition, as a categorical variable, high NLR (>2.75) was also correlated 
Table 5 The association of hematologic parameters with the patients' OS analysis

\begin{tabular}{|c|c|c|c|c|c|}
\hline Parameters & Median OS (months) & Number of patients & $P$ value & $\mathrm{HR}$ & $95 \% \mathrm{Cl}$ \\
\hline ANC $\leq 5,100$ & 10.9 & 68 & & & \\
\hline ALC $>1,819$ & 7.6 & 69 & 0.19 & 0.80 & $0.55-1.1$ \\
\hline$A L C \leq 1,819$ & 6.7 & 66 & & & \\
\hline $\mathrm{AMC} \leq 603$ & 7.8 & 68 & & & \\
\hline NLR $>2.75$ & 6.2 & 67 & 0.016 & 1.5 & $1.1-2.2$ \\
\hline$N L R \leq 2.75$ & 12.2 & 68 & & & \\
\hline MLR $>0.32$ & 6.3 & 68 & 0.36 & 1.2 & $0.83-1.7$ \\
\hline PLR $\leq 0.16$ & 10.9 & 67 & & & \\
\hline
\end{tabular}

OS, overall survival; HR, hazard ratio; CI, confidence interval; ANC, absolute neutrophil count; ALC, absolute lymphocyte count; AMC, absolute monocyte count; NLR, neutrophil-lymphocyte ratio; MLR, monocyte-lymphocyte ratio; PLR, platelet-lymphocyte ratio.

with negative OS outcome as shown in Table 6. However, baseline AMC $(>603 / \mu \mathrm{L}), \operatorname{AEC}(>147.5 / \mu \mathrm{L}), \operatorname{MLR}(>0.32)$, and ALC $(>1,819 / \mu \mathrm{L})$ were not shown to be linked to OS outcome.

The potential clinical value for associating high NLR with detecting distant metastases in PGL patients could possibly be explained by the pro-tumor nature of neutrophils (N2 phenotype). Neutrophils promote tumor proliferation and metastases via recruitment by cytokines, e.g., IL-17 and transforming growth factor beta (TGF- $\beta$ ), pro-angiogenic chemokines such as CXCL5 (epithelial neutrophil-activating peptide-78) (17), in addition to extracellular matrix-remodeling metalloproteases including MMP-9 (19) in the tumor microenvironment. High levels of circulating pro-tumor (N2 phenotype) neutrophils and subsequent neutrophilia can also promote tumor proliferation, in a cytokine-dependent manner, by inhibiting the activity of cytotoxic T-cells and inducing lymphocytopenia $(17,28-30)$.

Contrary to the pro-tumor neutrophils, circulating antitumor lymphocytes, such as $\mathrm{CD} 8^{+} \mathrm{T}$ cells, play an active role in hindering tumor progression and eradicating cancer cells through targeted cytotoxic cell-death (20). TGF- $\beta$, an immunosuppressive cytokine, has inhibitory effects on the activity of $\mathrm{CD} 8^{+} \mathrm{T}$ cells and macrophages in the tumor microenvironment (30-32). In addition, TGF- $\beta$ has a pro-tumor effect through its promotion of neutrophils' polarization towards the pro-tumor $(\mathrm{N} 2)$ phenotype and the recruitment of this specific phenotype (30). Utilizing TGF- $\beta$ blockers in immunotherapy was shown to have a promising anti-tumor action through enhancing the activation of $\mathrm{CD}^{+} \mathrm{T}$ cells, macrophages as well as promoting the anti-tumor (N1 phenotype) neutrophils $(30,33)$. Therefore, dysregulation of neutrophils and lymphocytes could explain the relationship between concurrent high NLR and low ALC, in support of our findings. Such a plausible explanation could be correlated to a higher incidence of distant metastases, possibly due to a diminished lymphocyte-mediated adaptive immune response to tumor cells (29). A hypothetical representation of the peripheral immune cells' association with distant metastases is shown in Figure 4.

Several studies reported the correlation of NLR with poor prognosis in multiple cancer types, including gastric and pancreatic cancer, with NLR cutoff values ranging from 2.3 to 5 , and sample sizes ranging from 65 to 502 patients $(12,14,34)$. The variation in the NLR value reported could be attributed to the varying demographic characteristics of the patients, in addition to their race $(29,35)$ as well as to possible concurrent infections.

Increased seeding of heterogeneous $\mathrm{CD}^{+} \mathrm{T}$ cells, which can have both pro- and anti-tumor roles, were associated 


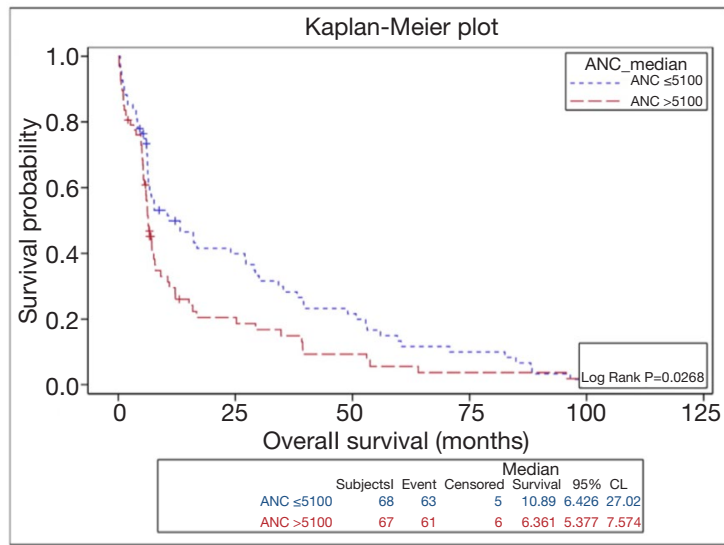

Figure 1 Kaplan-Meier curve for OS of PGL patients with ANC $>5,100$. OS, overall survival; PGL, primary gastric lymphoma; ANC, absolute neutrophil count; CI, confidence interval.

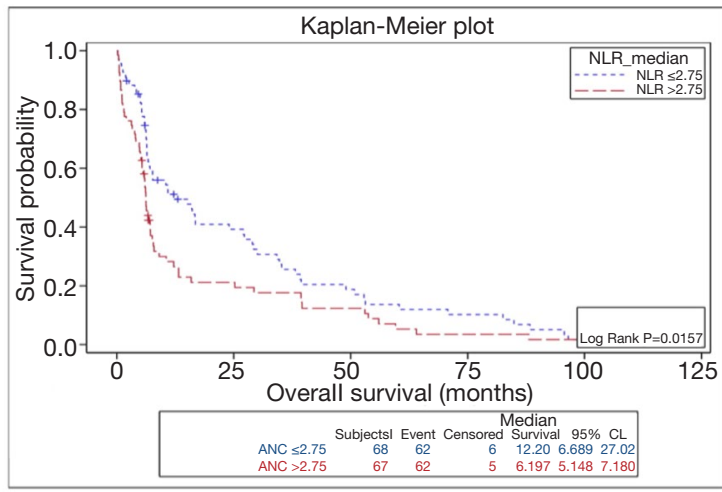

Figure 2 Kaplan-Meier curve for OS of PGL patients with NLR >2.75. OS, overall survival; PGL, primary gastric lymphoma; NLR, neutrophil-lymphocyte ratio; CI, confidence interval.

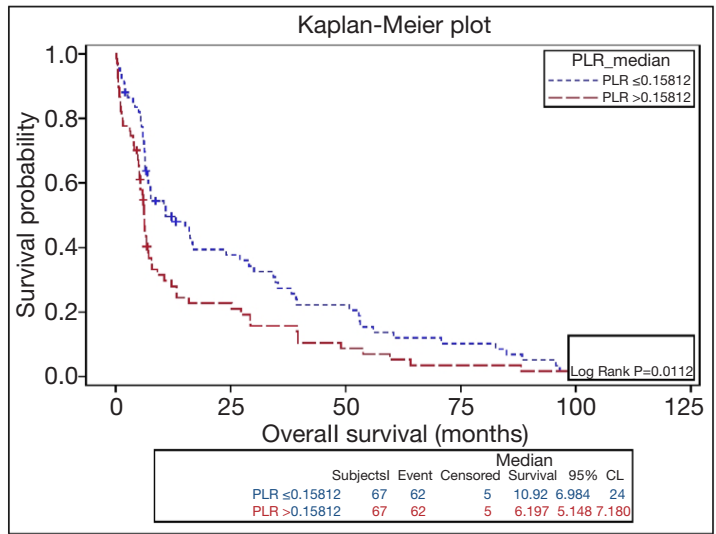

Figure 3 Kaplan-Meier curve for OS of PGL patients with PLR $>0.16$. OS, overall survival; PGL, primary gastric lymphoma; PLR, platelet-lymphocyte ratio; CI, confidence interval. with favorable outcomes in non-small cell lung cancers (NSCLCs) (36) but not in renal cell carcinoma (37). Despite not being significantly associated with survival outcomes in PGL patients; monocyte counts correlated with poor survival outcomes in pancreatic cancer (38). Pro-tumor monocytes can suppress T-cell infiltration and cytotoxic activity $(24,39)$. In addition, inflammatory monocytes are associated with poor survival in lung squamous carcinomas (LUSC) through inducing fibrin cross-linking, thus remodeling the extracellular-matrix and promoting tumor progression (40).

Treatment plans to limit distant metastases in MALT lymphoma, the most common type of indolent gastric lymphoma, include eradication of $H$. pylori as first-line therapy, in addition to chemotherapy, radiation, and immunotherapy $(41,42)$, the latter can be promising. In this aspect, evaluating hematological markers, especially neutrophils with their contribution to a high NLR and concurrent lower $\mathrm{CD} 8^{+} \mathrm{T}$ cells, immunotherapy can be of special interest in PGL. Immunotherapy has shown promising results through specifically blocking cytokines and pro-angiogenic factors that recruit and induce protumor polarization of neutrophils $(17,30)$. These targets include blocking of IL-17, G-CSF, TGF- $\beta$, VEGF, and IL-8 $(17,18,30,43,44)$. Immunotherapy with rituximab, an anti-CD20 antibody, was shown to achieve complete MALT lymphoma remission of $46 \%$ (45).

Immunotherapy has made a significant impact on the management of various cancers (46). NLR correlates with the successful response to chemotherapy and immunotherapy in various cancers $(47,48)$. Future research highlights the possibility of further exploring the relationship of NLR with the response of distant metastases to various treatment modalities. In the view of the increasingly-challenging immune-related adverse events (irAEs) caused by immunotherapy (49), a breakthrough achievement in the management of irAEs can be attained if NLR could identify patients at a higher risk for developing irAEs.

Potential statistical restraints in this study involve the retrospective nature of the collected information as well as the utilization of the ROC analysis, which is sample-dependent and can vary across studies. Additionally, as all data was gathered from the same institution, this could bring referral and management bias as one of the limitations. Although confounding factors like prior steroid administration were excluded from this patient cohort, other possible inflammatory responses, including concurrent infections, cannot be entirely 
Table 6 The association between NLR, as a categorical variable, and OS

\begin{tabular}{lccc}
\hline NLR & OS (months) & HR & $95 \% \mathrm{Cl}$ \\
\hline$<1.69$ & 14.2 & Reference & \\
$1.69-2.75$ & 10.9 & 1.1 & $0.68-1.9$ \\
$2.75-4.59$ & 6.2 & 1.7 & $1.03-2.8$ \\
$>4.59$ & 6.1 & 1.6 & $0.97-2.6$ \\
\hline
\end{tabular}

NLR, neutrophil-lymphocyte ratio; OS, overall survival; HR, hazard ratio; Cl, confidence interval.

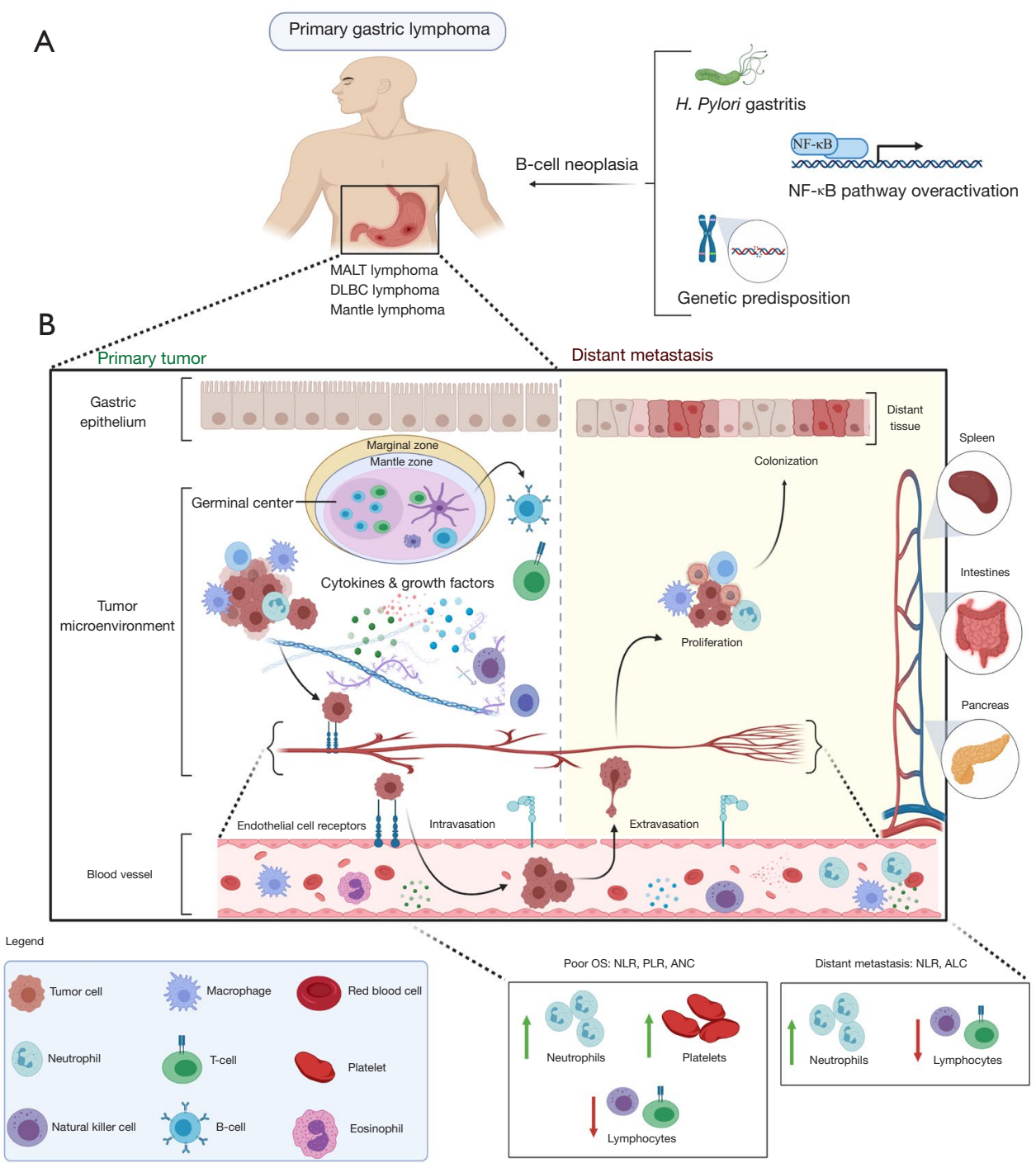

Figure 4 (A) A hypothesized pathway depicting the etiological factors of PGL, being mostly mediators of B-cell neoplasia; (B) a hypothetical representation of the peripheral immune cell counts' association with distant metastases starting from the primary tumor site (e.g., stomach cardia, fundus). PGL includes but is not limited to low-grade marginal zone lymphoma (known as MALT lymphoma), mantle zone lymphoma, in addition to high-grade DLBCL of the germinal center. High NLR and low ALC in circulation were associated with baseline distant metastases. High baseline ANC, NLR, and PLR were associated with poor OS. PGL, primary gastric lymphoma; MALT, mucosa-associated lymphoid tissue; DLBCL, diffuse large B-cell lymphoma; ANC, absolute neutrophil count; NLR, neutrophil-lymphocyte ratio; PLR, platelet-lymphocyte ratio; OS, overall survival. 
ruled out. Future analyses can include further clinical variables that can potentially impact clinical outcomes in PGL. Despite being limited with a small sample size of 139 PGL patients due to the rarity of PGL, nevertheless, we aimed to explore the clinical value of different inflammatory cell counts, specifically in relation to the incidence of higher baseline distant metastases in PGL patients.

\section{Conclusions}

In PGL patients, high baseline NLR and ALC can be considered as predictive parameters for the status of baseline distant metastases. Elevated baseline NLR, PLR and ANC were associated with poor OS. The hematologic markers investigated in this study, especially NLR, might be potentially helpful to assess and correlate with the response success to treatment in PGL.

\section{Acknowledgments}

The authors appreciate Mrs. Ayat Taqash's assistance in conducting statistical analysis. Figure 4 was created using biorender.com.

Funding: None.

\section{Footnote}

Reporting Checklist: The authors have completed the REMARK reporting checklist. Available at http://dx.doi. org/10.21037/jgo-20-383

Data Sharing Statement: Available at http://dx.doi. org/10.21037/jgo-20-383

Conflicts of Interest: All authors have completed the ICMJE uniform disclosure form (available at http://dx.doi. org/10.21037/jgo-20-383). The authors have no conflicts of interest to declare.

Ethical Statement: The authors are accountable for all aspects of the work in ensuring that questions related to the accuracy or integrity of any part of the work are appropriately investigated and resolved. The study was conducted in accordance with the Declaration of Helsinki (as revised in 2013). Approval to conduct this study was obtained through the IRB Office at King Hussein Cancer Center (IRB Approval No. 18KHCC54). Individual consent for this retrospective analysis was waived.
Open Access Statement: This is an Open Access article distributed in accordance with the Creative Commons Attribution-NonCommercial-NoDerivs 4.0 International License (CC BY-NC-ND 4.0), which permits the noncommercial replication and distribution of the article with the strict proviso that no changes or edits are made and the original work is properly cited (including links to both the formal publication through the relevant DOI and the license). See: https://creativecommons.org/licenses/by-nc-nd/4.0/.

\section{References}

1. Zucca E, Cavalli F. Extranodal lymphomas. Ann Oncol 2000;11:219-22.

2. Zullo A, Hassan C, Ridola L, et al. Gastric MALT lymphoma: old and new insights. Ann Gastroenterol 2014;27:27-33.

3. Zucca E, Roggero E, Bertoni F, et al. Primary extranodal non-Hodgkin's lymphomas. Part 1: Gastrointestinal, cutaneous and genitourinary lymphomas. Ann Oncol 1997;8:727-37.

4. Ghimire P, Wu GY, Zhu L. Primary gastrointestinal lymphoma. World J Gastroenterol 2011;17:697-707.

5. Psyrri A, Papageorgiou S, Economopoulos T. Primary extranodal lymphomas of stomach: clinical presentation, diagnostic pitfalls and management. Ann Oncol 2008;19:1992-9.

6. Kawahara Y, Mizuno M, Yoshino T, et al. HLADQA1*0103-DQB1*0601 haplotype and Helicobacter pylori-positive gastric mucosa-associated lymphoid tissue lymphoma. Clin Gastroenterol Hepatol 2005;3:865-8.

7. Bertoni F, Zucca E. Delving deeper into MALT lymphoma biology. J Clin Invest 2006;116:22-6.

8. Hussell T, Isaacson PG, Crabtree JE, et al. The response of cells from low-grade B-cell gastric lymphomas of mucosa-associated lymphoid tissue to Helicobacter pylori. Lancet 1993;342:571-4.

9. Munari F, Lonardi S, Cassatella MA, et al. Tumorassociated macrophages as major source of APRIL in gastric MALT lymphoma. Blood 2011;117:6612-6.

10. Darom A, Gomatos IP, Leandros E, et al. Molecular markers (PECAM-1, ICAM-3, HLA-DR) determine prognosis in primary non-Hodgkin's gastric lymphoma patients. World J Gastroenterol 2006;12:1924-32.

11. Grivennikov SI, Greten FR, Karin M. Immunity, inflammation, and cancer. Cell 2010;140:883-99.

12. Abu-Shawer O, Abu-Shawer M, Shurman A, et al. The clinical value of peripheral immune cell counts in 
pancreatic cancer. PLoS One 2020;15:e0232043.

13. Zhang X, Zhang W, Feng LJ. Prognostic significance of neutrophil lymphocyte ratio in patients with gastric cancer: a meta-analysis. PloS one 2014;9:e111906.

14. Abu-Shawer O, Abu-Shawer M, Haimour A, et al. Hematologic markers of distant metastases in gastric cancer. J Gastrointest Oncol 2019;10:529-36.

15. Abu-Shawer M, Abu-Shawer O, Souleiman M, et al. Hematologic markers of lung metastasis in stage IV colorectal cancer. J Gastrointest Cancer 2019;50:428-33.

16. Shen M, Hu P, Donskov F, et al. Tumor-associated neutrophils as a new prognostic factor in cancer: a systematic review and meta-analysis. PLoS One 2014;9:e98259.

17. Masucci MT, Minopoli M, Carriero MV. Tumor associated neutrophils. their role in tumorigenesis, metastasis, prognosis and therapy. Front Oncol 2019;9:1146.

18. Coffelt SB, Kersten K, Doornebal CW, et al. IL-17producing $\gamma \delta \mathrm{T}$ cells and neutrophils conspire to promote breast cancer metastasis. Nature 2015;522:345-8.

19. Deryugina EI, Zajac E, Juncker-Jensen A, et al. Tissueinfiltrating neutrophils constitute the major in vivo source of angiogenesis-inducing MMP-9 in the tumor microenvironment. Neoplasia 2014;16:771-88.

20. Kitamura T, Qian BZ, Pollard JW. Immune cell promotion of metastasis. Nat Rev Immunol 2015;15:73-86.

21. DeNardo DG, Andreu P, Coussens LM. Interactions between lymphocytes and myeloid cells regulate proversus anti-tumor immunity. Cancer Metastasis Rev 2010;29:309-16.

22. Puxeddu I, Alian A, Piliponsky AM, et al. Human peripheral blood eosinophils induce angiogenesis. Int J Biochem Cell Biol 2005;37:628-36.

23. Kisucka J, Butterfield CE, Duda DG, et al. Platelets and platelet adhesion support angiogenesis while preventing excessive hemorrhage. Proc Natl Acad Sci USA 2006;103:855-60.

24. Olingy CE, Dinh HQ, Hedrick CC. Monocyte heterogeneity and functions in cancer. J Leukoc Biol 2019;106:309-22.

25. Schmall A, Al-Tamari HM, Herold S, et al. Macrophage and cancer cell cross-talk via CCR2 and CX3CR1 is a fundamental mechanism driving lung cancer. Am J Respir Crit Care Med 2015;191:437-47.

26. Shoenfeld Y, Gurewich Y, Gallant LA, et al. Prednisoneinduced leukocytosis. Influence of dosage, method and duration of administration on the degree of leukocytosis. Am J Med 1981;71:773-8.
27. Nakagawa M, Terashima T, D'Yachkova Y, et al. Glucocorticoid-induced granulocytosis: contribution of marrow release and demargination of intravascular granulocytes. Circulation 1998;98:2307-13.

28. Fridlender ZG, Albelda SM, Granot Z. Promoting metastasis: neutrophils and $T$ cells join forces. Cell Res 2015;25:765-6.

29. Howard R, Kanetsky PA, Egan KM. Exploring the prognostic value of the neutrophil-to-lymphocyte ratio in cancer. Sci Rep 2019;9:19673.

30. Fridlender ZG, Sun J, Kim S, et al. Polarization of tumorassociated neutrophil phenotype by TGF-beta: "N1" versus "N2" TAN. Cancer Cell 2009;16:183-94.

31. Ge R, Rajeev V, Ray P, et al. Inhibition of growth and metastasis of mouse mammary carcinoma by selective inhibitor of transforming growth factor-beta type I receptor kinase in vivo. Clin Cancer Res 2006;12:4315-30.

32. Tsunawaki S, Sporn M, Ding A, et al. Deactivation of macrophages by transforming growth factor-beta. Nature 1988;334:260-2.

33. Suzuki E, Kim S, Cheung HK, et al. A novel smallmolecule inhibitor of transforming growth factor beta type I receptor kinase (SM16) inhibits murine mesothelioma tumor growth in vivo and prevents tumor recurrence after surgical resection. Cancer Res 2007;67:2351-9.

34. Yang JJ, Hu ZG, Shi WX, et al. Prognostic significance of neutrophil to lymphocyte ratio in pancreatic cancer: a meta-analysis. World J Gastroenterol 2015;21:2807-15.

35. Hsieh M, Chin K, Link B, et al. Benign ethnic neutropenia in individuals of African descent: incidence, granulocyte mobilization, and gene expression profiling. Blood 2005;106:3069.

36. Wakabayashi O, Yamazaki K, Oizumi S, et al. CD4+ T cells in cancer stroma, not CD8+ T cells in cancer cell nests, are associated with favorable prognosis in human non-small cell lung cancers. Cancer Sci 2003;94:1003-9.

37. Siddiqui SA, Frigola X, Bonne-Annee S, et al. Tumorinfiltrating Foxp3-CD4+CD25+ T cells predict poor survival in renal cell carcinoma. Clin Cancer Res 2007;13:2075-81.

38. Sanford DE, Belt BA, Panni RZ, et al. Inflammatory monocyte mobilization decreases patient survival in pancreatic cancer: a role for targeting the CCL2/CCR2 axis. Clin Cancer Res 2013;19:3404-15.

39. Gallina G, Dolcetti L, Serafini P, et al. Tumors induce a subset of inflammatory monocytes with immunosuppressive activity on CD8+ T cells. J Clin Invest 2006;116:2777-90. 
40. Porrello A, Leslie PL, Harrison EB, et al. Factor XIIIAexpressing inflammatory monocytes promote lung squamous cancer through fibrin cross-linking. Nat Commun 2018;9:1988.

41. Zullo A, Hassan C, Ridola L, et al. Eradication therapy in Helicobacter pylori-negative, gastric low-grade mucosaassociated lymphoid tissue lymphoma patients: a systematic review. J Clin Gastroenterol 2013;47:824-7.

42. Zucca E, Dreyling M. Gastric marginal zone lymphoma of MALT type: ESMO Clinical Practice Guidelines for diagnosis, treatment and follow-up. Ann Oncol 2010;21:v175-6.

43. Huh SJ, Liang S, Sharma A, et al. Transiently entrapped circulating tumor cells interact with neutrophils to facilitate lung metastasis development. Cancer Res 2010;70:6071-82.

44. Nakamura M, Takahashi T, Matsui H, et al. New pharmaceutical treatment of gastric MALT lymphoma: antiangiogenesis treatment using VEGF receptor antibodies and celecoxib. Curr Pharm Des 2014;20:1097-103.

Cite this article as: Haimour A, Abu-Shawer O, Abu-Shawer M, Al-Taji A, Altamimi T, Mansour R, Amarin JZ, Sultan H, Al-Hussaini M. The clinical potential of circulating immune cell counts in primary gastric lymphoma. J Gastrointest Oncol 2021;12(2):365-376. doi: 10.21037/jgo-20-383
45. Martinelli G, Laszlo D, Ferreri AJ, et al. Clinical activity of rituximab in gastric marginal zone non-Hodgkin's lymphoma resistant to or not eligible for anti-Helicobacter pylori therapy. J Clin Oncol 2005;23:1979-83.

46. Abu-Shawer O, Bushnaq T, Abu-Shawer M. Cancer immunotherapy: an updated overview of current strategies and therapeutic agents. Gulf J Oncolog 2019;1:76-82.

47. Liu D, Jin J, Zhang L, et al. The neutrophil to lymphocyte ratio may predict benefit from chemotherapy in lung cancer. Cell Physiol Biochem 2018;46:1595-605.

48. Khunger M, Patil PD, Khunger A, et al. Post-treatment changes in hematological parameters predict response to nivolumab monotherapy in non-small cell lung cancer patients. PLoS One 2018;13:e0197743.

49. Abu-Shawer O, Singh P, Yenulevich E, et al. Novel platform leveraging electronic medical record (EMR) to triage patients admitted with high-grade immune-related adverse events (irAEs) to the immune-toxicity (ITOX) service. J Immunother Cancer 2020;8:e000992. 


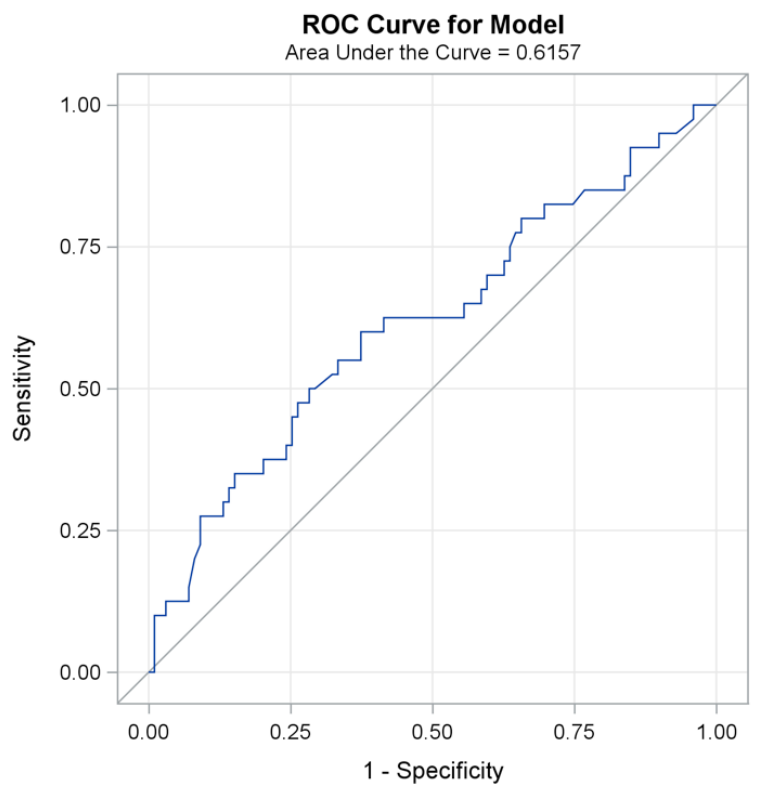

Figure S1 ROC curve showing the AUC for determining NLR. ROC, receiver operating characteristic; AUC, area under the curve; NLR, neutrophil-lymphocyte ratio. 